\title{
Characterization of Fault Recovery through Fault Injection on FTMP
}

\author{
George B. Finelli \\ NASA Langley Research Center, Hampton
}

\begin{abstract}
Key Words-Fault detection, Fault injection, Fault recovery, Parameter estimation, Reliability modeling, Sampling methods, Statistical distributions.

Reader Aids-

Purpose: To present a model and methods for using it Special math needed for explanations: Basic statistics Special math needed to use results: None Results useful to: Reliability analysts, system designers
\end{abstract}

Abstract-The statistical methods used to collect and analyze fault-recovery data affect directly the credibility of reliability estimation. To provide data on which to base the development of sampling methods and parameter estimation techniques, pin-level fault-injection was conducted on the FTMP computer. Detection time was chosen for statistical analysis because it accounted for most of the variation in total recovery time. Stuck-at-zero, stuckat-one, and inverted faults were injected on each of six pins, yielding 18 data sets. The data sets fell into groups of detection behavior; however, none of the factors that were varied in the experiment - fault type, pin, chip, or board - acounted for the groupings. While no single distribution was shown to be the best fit to all the data sets, of greater importance is that the exponential distribution was a bad fit to all data sets. This refutes a common assumption of reliability modeling that detection times are exponentially distributed. These results suggest that stratified random sampling methods and statistically robust parameter estimation techniques are required to characterize fault detection time. Further experimentation is planned to discover the sources of the variation in detection time.

\section{INTRODUCTION}

To achieve the high levels of reliability required for advanced flight-control, on-board digital computers must incorporate fault-recovery which enables them to continue functioning despite the occurrence of faults. Fault recovery involves three separate events: detection of a fault, identification of the faulty unit, and reconfiguration of system resources to render the effects of the fault harmless to the operation of the system. The feasibility of fault-tolerant computers suitable for real-time applications has been demonstrated by the implementation of two test specimens, the Fault-Tolerant Multiprocessor (FTMP) [1] and the Software Implemented Fault Tolerance (SIFT) computer [2].

Verifying that systems are consistent with their intended designs and validating that they are suitable for specific applications are critical steps in the general acceptance and widespread use of fault-tolerant computers in aerospace applications. Due to potential life-threatening situations imposed by system failure of on-board computers performing active flight control, reliability estimation has received highest priority in the development of methods for verification and validation.

Due to long mean-times-between-failures and the high cost of systems like FTMP and SIFT, the classical validation approach of life testing is impractical for estimating the reliability of fault-tolerant systems. Therefore, modeling techniques must be used which account for both faultoccurrence behavior and fault-recovery mechanisms. The approach currently used to provide the input data needed to model reliability is to predict the parameters related to the fault-occurrence processes from failure history or through Part Stress and Part Count methods, such as those used in US Military Handbook 217D [3], and to estimate the parameters related to fault recovery using data generated from fault-injection experimentation.

Developing assessment and validation techniques for fault-tolerant systems is a major objective of the reserch being conducted and funded at the NASA Langley Reserch Center's AIRLAB facility [4]. These techniques will be incorporated into comprehensive design and validation methodologies for systems targeted for advanced aerospace applications. Validation of fault-tolerant avionics and control systems is discussed extensively by many authors in [5] and specific validation tasks are identified in [6].

The objective of the work described in this paper is to develop fault-injection procedures and statistical analysis techniques to characterize the fault recovery of faulttolerant systems. Because relatively little data are available in the literature, faults were injected into FTMP in AIRLAB to generate data to assess the utility of current faultinjection sampling methods and the validity of common reliability-modeling assumptions concerning the statistical distribution of recovery times.

\section{FAULT INJECTION EXPERIMENTATION}

Paramount to the validation of fault-tolerant systems is reliability estimation. Essential steps in establishing the reliability of fault-tolerant computers are verification of fault-handling mechanisms and characterization of their behavior. Fault injection can be used to verify the operation of fault-handling mechanisms, while characterization requires statistical analysis of the data generated from this type of experimentation. In order to obtain meaningful data in reasonable periods of time, experiments must be carefully designed. In particular, appropriate sampling methods must be formulated. To make optimal use of the generated data, 
the underlying distributions of the recovery processes must be established. This distributional information affects the choice of the assumptions on which reliability models are based and the techniques used to estimate input parameters for these models.

A number of sampling methods are being used in fault injection, each with strengths and weaknesses. The surest way of characterizing a system is to do exhaustive testing. Obviously, for digital systems which are highly complex and redundant, such testing can be too time consuming and costly. Simple random sampling can cut down the amount of testing, but the price may be unacceptably large variations and uncertainty in parameter estimates if there are some underlying factors which affect recovery. Sampling proportional to failure rate is also used, but this technique is based on the fault occurrence processes and does not account for a system's ability to recover from faults based on the recovery mechanisms themselves.

Once data have been generated from properly designed experiments, parameter estimation techniques must be employed to produce the fault recovery input values for reliability analysis. Parameter estimation depends on the sampling method used to generate the data and the underlying distribution of the data. A common assumption made in reliability modeling is that fault recovery times are exponentially distributed. This simplifies parameter estimation to computation of the sample mean. Other distributions, like the Weibull and gamma, can require iterative solution of maximum-likelihood equations or some type of linear estimation technique to get $s$-efficient estimators [7].

Designing experiments and choosing appropriate estimation techniques require some knowledge of the system under test and the behavior which must be characterized. Researchers at the Charles Stark Draper Laboratory injected faults into the FTMP to verify the system fault-handling capabilities and the parameter values that they assumed in their reliability analysis [8]. In their final analysis, all the data were grouped together to get an overall picture of recovery behavior. While this built confidence in the system, there was no attempt to identify variation in behavior other than at the circuit board level or to account for possible variation in behavior in the estimation of system-level parameters.

The objective of the work described in this paper was not to validate FTMP, but to provide a statistical basis for conducting fault-injection experiments on fault-tolerant computers. Also, the validity of exponential recovery times was investigated.

\section{FTMP and the Fault-Injection Environment}

The FTMP was designed to achieve extremely high levels of reliability suitable for advanced aerospace applications. The engineering test specimen resident in AIRLAB was implemented to meet the requirements of flight-critical fly-by-wire flight control for advanced civil transport aircraft. For this application, a redundancy level of 10 was chosen; 10 nominally identical Line Replaceable Units (LRUs), each containing a processor region, slave region, clock generation region, power subsystem, two bus guardian units, and system bus circuits [9].

From an application programmer's point of view, the FTMP is a multiprocessor with three virtual processors. In actuality, each virtual processor of the multiprocessor is a processor triad - three processors operating identical software in tight synchronization and voting on all data read from or written to system memory. This is how FTMP achieves its fault tolerance and computation integrity. A single failure in any processor of a triad can be handled without erroneous computations propagating outside the FTMP.

The processor triads access a single shared memory, 10 input/output ports, a real time clock/counter, and status/control registers. The memory elements in each LRU are also grouped into triads, with each triad being a section of the system's single shared memory.

During normal operation, within a processor triad, the results of each input/output operation are voted. If a disagreement is found, information concerning which processor in the triad is faulty is written to error latches. An executive software program called the System Configuration Controller (SSC) reads the error latches, and then triggers the appropriate identification and reconfiguration actions.

\section{Fault-Injection Procedure}

The capability to inject faults into a fault-tolerant system like FTMP is an important part of the reliability estimation and the overall validation of such systems. Therefore, as part of the FTMP experimentation setup, a hardware fault injector, interface hardware, and support software were built by Draper Laboratory to enable fault injection. The hardware of FTMP has been modified to support fault injection. LRU \#3 is specially constructed for connection of the fault injector. The side of FTMP's rack has a door which opens to expose LRU \#3. The boards of LRU $\# 3$ can be extended so the chips are accessible. All chips in this LRU are connected to sockets which allow them to be removed for insertion of the fault injector implant. Since all LRUs are nominally identical and the system architecture is symmetrical with respect to LRUs, it is assumed that injecting faults into the same LRU repeatedly will provide data indicative of faults occurring in any LRU.

The FTMP executive software has been modified to support fault injection. A speical version of the SCC, called FSCC, runs on the system during fault injection. This program controls the internal state of FTMP at the time of injection and provides communication of relevant data to the host system.

The FTMP fault-injection environment and process are shown in figure 1 . The Draper fault-injector implant and fault-injection software have been modified to interface 
with AIRLAB resources. The fault-injection process is hosted by a VAX 11/750, with commands and data being transferred across a 1553/UNIBUS Direct Memory Access (DMA) board. The fault injection software, running on the VAX, accepts commands from a terminal and controls the fault injection process. The process starts with a get ready command from the VAX. The FTMP acknowledges and restores LRU \#3 to a fault-free state. After a fault is injected, the FTMP writes the set of data to the DMA board which in turn writes it to VAX memory for the VAX to read.

With the test environment as described, pin-level fault injection can be conducted automatically. The procedure requires that the experimenter construct a command file on the host VAX, which can then be invoked to start a test. The fault injector can be connected to up to 48 pins on one chip or multiple chips.

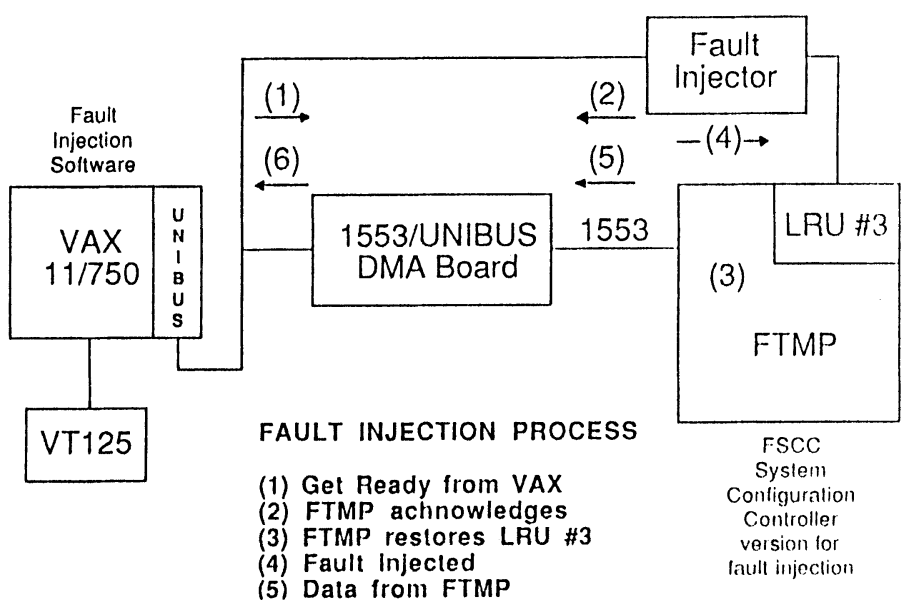

Fig. 1. FTMP fault injection and support environment.

\section{Statistical Design of the Experiment}

A common approach to obtaining and using data for reliability analysis is to conduct testing, assume the data follow the distributional form on which the reliability model is based, and estimate the parameters accordingly. When the behavior to be parameterized is fault recovery, the testing procedure of choice is fault injection. Even with automatic capabilities, exhaustive fault injection is a costly and time consuming process.

To construct appropriate sampling methods to reduce the time and cost of testing, knowledge must be obtained about the nature of fault recovery behavior. One of the goals of this effort was to learn enough about fault recovery, using FTMP as a test specimen, to formulate test guidelines for fault-tolerant systems in general.

Another goal was to establish the distributions of recovery time and its components - detection, identification, and reconfiguration. This information, combined with knowledge of the sampling methods used to generate the data, will enable selection of proper parameter estimation techniques. While parameter values for FTMP were computed, these specific values were not the most important results. The prime concerns of this effort were the lessons which could be learned about fault recovery and fault injection in general.

Figure 2 shows the layout of the experiment designed to achieve these goals. In this experiment, three chips were selected from two boards. The first board in the diagram is the Cache Controller. On this board, a quadruple 2-input positive-NOR gate chip (54LS02) and a dual 4-input positive-NAND gate chip (54LS20), were selected. The second board is one of two Central Processing Unit boards found in each LRU. The chip selected from this board was a quadruple data selector/multiplexer chip (54LS257). For each chip, two pins were selected for injection of stuck-atzero, stuck-at-one, and inverted faults. This experiment produced 18 data sets, as represented by the boxes with index numbers at the bottom of figure 2 . To generate each data set, $\mathbf{6 0 0}$ faults were injected. Summary statistics for each data set are presented in table 1 . The last 4 columns of table 1 show estimates of the scale and shape parameters for the Weibull and gamma distributions. The relevance of this information is discussed in section 3. Two statistical analyses were conducted on the 18 data sets.

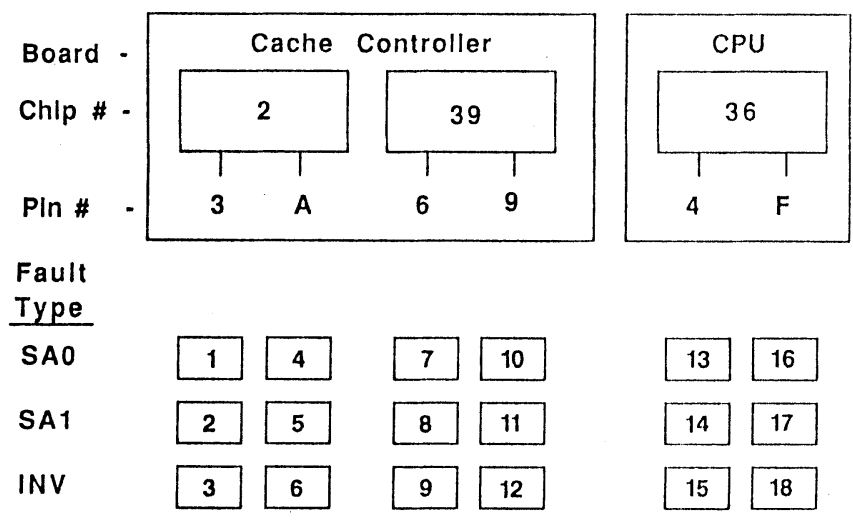

Fig. 2. Design of experiment - injection locations and fault types.

A. Multiple comparisons. The objectives of this analysis were to discover any possible variation in behavior from one data set to another and to identify the sources of the variation. For example, a single pin may behave the same regardless of the type of fault injected, or it may behave differently for each. Or, for a particular fault type, all pins may exhibit similar behavior. The reason for this type of analysis is to provide the information required to formulate appropriate sampling methods. The discriminator for appropriateness is $s$-efficiency; that is, the sampling method should minimize time and cost of the test while keeping the variance of the estimators based on the data to a minimum.

The best situation that could be anticipated is for all data sets to show the same behavior. In other words, regardless of the fault type or pin location, the distribution of the recovery times is the same. In this case, simple random sampling is the most $s$-efficient method. However, if 
TABLE 1

Summary Statistics for Detection Time Data Sets

(All times are in milliseconds)

\begin{tabular}{rccccccccc}
\hline & & & & & & \multicolumn{2}{c}{ Weibull } & \multicolumn{2}{c}{ Gamma } \\
Set & $\begin{array}{c}\text { Sample } \\
\#\end{array}$ & Size & Minimum & Maximum & $\begin{array}{c}\text { Sample } \\
\text { Mean }\end{array}$ & $\begin{array}{c}\text { Standard } \\
\text { Deviation }\end{array}$ & $\begin{array}{c}\text { Parameters } \\
\text { Scale }\end{array}$ & Shape & Parameters \\
\hline 1 & 600 & 80 & 1002 & 311.7 & 114.2 & 349.2 & 2.93 & 46.39 & 6.72 \\
2 & 600 & 74 & 511 & 262.9 & 95.06 & 294.6 & 3.07 & 39.68 & 6.63 \\
3 & 600 & 73 & 571 & 266.9 & 94.44 & 298.7 & 3.16 & 38.93 & 6.86 \\
4 & 600 & 75 & 883 & 302.8 & 111.8 & 339.4 & 2.93 & 46.59 & 6.50 \\
5 & 600 & 80 & 1148 & 302.4 & 116.6 & 339.1 & 2.70 & 48.11 & 6.29 \\
6 & 600 & 83 & 9753 & 1874. & 1970. & 1824. & .946 & 1969. & .952 \\
7 & 600 & 73 & 944 & 322.5 & 139.9 & 364.5 & 2.47 & 61.39 & 5.25 \\
8 & 600 & 61 & 775 & 300.3 & 140.9 & 339.9 & 2.29 & 72.56 & 4.14 \\
9 & 600 & 75 & 536 & 261.5 & 98.66 & 293.7 & 2.94 & 43.01 & 6.08 \\
10 & 599 & 67 & 840 & 266.2 & 118.5 & 300.8 & 2.40 & 55.46 & 4.80 \\
11 & 600 & 71 & 866 & 309.1 & 144.8 & 349.8 & 2.28 & 71.38 & 4.33 \\
12 & 599 & 73 & 511 & 249.0 & 98.17 & 280.2 & 2.82 & 46.28 & 5.38 \\
13 & 600 & 69 & 508 & 253.2 & 92.38 & 283.9 & 3.08 & 39.70 & 6.38 \\
14 & 599 & 73 & 513 & 245.6 & 93.92 & 276.1 & 2.90 & 41.29 & 5.95 \\
15 & 600 & 69 & 474 & 247.8 & 91.23 & 277.9 & 3.02 & 38.97 & 6.36 \\
16 & 598 & 35 & 906 & 339.8 & 181.5 & 384.2 & 1.98 & 111.1 & 3.06 \\
17 & 600 & 34 & 839 & 333.2 & 180.0 & 376.5 & 1.95 & 113.1 & 2.95 \\
18 & 600 & 71 & 481 & 242.1 & 94.70 & 272.4 & 2.84 & 43.48 & 5.57 \\
\hline
\end{tabular}

there is variation from data set to data set, simple random sampling may introduce unacceptable amounts of variation into parameter estimates. When there is variation and grouping of behavior, stratified sampling is the best method. This method is based on the assumption that the behavior is stratified, which means the population under consideration can be divided into nonoverlapping groups (strata) [10].

B. Distribution fitting. The objective was to establish the statistical distribution which best fits the data. The distributions used as candidates were the exponential, Weibull, gamma, and lognormal. The exponential is of particular interest because it is commonly assumed in reliability modeling that fault recovery is distributed exponentially. Establishing the distribution of the data provides the basis for selecting parameter estimation techniques and for verifying the assumptions on which reliability models are based.

\section{RESULTS AND DISCUSSION}

Most of the variation in total recovery time comes from fault detection. As reported by Draper Laboratory, this behavior was anticipated. Therefore, detection time was chosen as the random variable for in-depth analysis. Fault-detection time was measured from the time a fault was injected at a pin of an integrated circuit chip to the time that the executive software read the error latches and discovered a miscompare from the voting process.

\section{Multiple Comparisons}

The multiple comparisons of the data sets were conducted through the use of box plots. The box plot is a graphical display that provides information about a sample frequency distribution [11]. The technique is particularly effective in displaying the behavior in the tails or extreme values of a distribution.

Basically, box plots display the median, quartiles, and outliers of a data set. The median is the middle value of a set of data and divides that set in half. Quartiles divide a set into quarters. The first or $25 \%$ quartile is the value at which $25 \%$ of the data points are less than or equal to that value. The median is the second quartile and the third or $75 \%$ quartile is the value at which $75 \%$ of the data points are less than or equal to that value. The interquartile range (IQR) is the distance between the first and third quartiles.

The IQR is used to determine which points are considered outliers. The IQR is multiplied by 1.5 and subtracted from the first quartile to get the lower inner fence and again subtracted from this value to get the lower outer fence. Similarly, this factor is added to the third quartile to get the upper inner fence and again added to this value to get the upper outer fence.

Figure 3 shows box plots of the data sets generated from the fault injection. In each box plot, the median is represented as the horizontal line across the middle of the box part of the plot. The bottom horizontal side of the box is the first quartile and the top horizontal side is the third quartile. The shorter horizontal line below the first quartile is the smallest data point which is greater than the lower inner fence. Likewise, the short horizontal line above the third quartile is the largest data point which is less than the upper inner fence. These two points are called the adjacent values. The mild outliers, represented by the dots, are the data points which fall between the upper adjacent value and the upper inner fence. The extreme outliers, represented by the 
crosses, are the points which are greater than the upper outer fence.

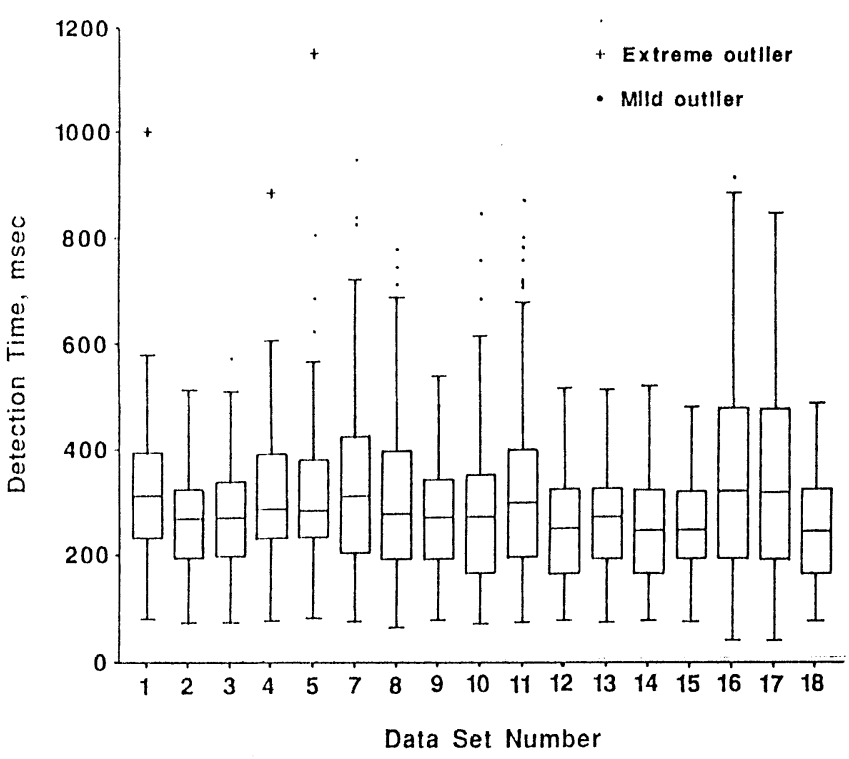

Fig. 3. Multiple comparisons through box plots.

By looking across the box plots, some results are obvious. The detection times fall into groups of behavior. There are four groups evident: the first group includes data sets 1, 4, 5; the second group includes sets 2, 3, 9, 12-15, 18; the third group includes sets $7,8,11$; and the fourth group includes sets 16,17 . By visual inspection and evaluation of the summary statistics, data set 10 can not be classified. It either belongs to one of the first three groups or it represents another distinct group. Data set 6 was not plotted because of its shift in scale. It is unique from the other data sets and represents a place where FTMP's fault detection mechanisms had trouble detecting the faults. The number of groups is not the important fact and this display is not to suggest these are all the groupings that would be seen if exhaustive testing were performed.

Two important conclusions are: 1) The detection behavior is not homogeneous across all data sets. This indicates that stratified sampling methods need to be developed for fault injection. 2) None of the factors under experimental control can account for the observed groupings of behavior. For each fault type, at least two pins behave differently. This can be seen by looking at every fourth plot. Also, for a single pin, the detection behavior varies for different fault types. The only case where this is not true is for data sets 13-15. Hence, appropriate sampling methods could not be formulated based on the data from this experiment.

\section{Distribution Fitting}

Hypothesis testing and graphical techniques were employed to infer the statistical distribution of detection time. Four distributions were chosen: exponential, Weibull, gamma, and lognormal. The chi-square test of goodness of fit was applied to each data set for each distribution; and each pdf was plotted against a histogram of each data set to provide visual support of the test results.

In conducting the chi-square test, the number of intervals which the data are broken into is at the discretion of the analyst [12]. The degrees of freedom for each test statistic were computed by subtracting the number of parameters in the null distribution plus one from the number of intervals used in the test. Along with the values of the test statistics, the corresponding $s$-critical levels were also computed. The $s$-critical level of a test is defined here as the smallest $s$-significance level at which the null hypothesis would be rejected for the given observations [13]. The s-significance level is the maximum probability of rejecting a true null hypothesis. Often, 0.05 or 0.01 is chosen as the $s$-significance level. By stating the $s$-critical level of the test, all tests of greater $s$-significance levels lead to rejection and all tests of smaller $s$-significance levels lead to non-rejection.

The results of the chi-square tests are summarized in table 2. For all data sets, the exponential distribution yielded zero or near-zero critical levels. Thus the detectiontime data are clearly not distributed according to the exponential distribution. Of the remaining three distributions, none was found to be the best fit to all the data sets. The gamma and the Weibull distributions were best for ten and six data sets respectively. The lognormal distribution was the best in one case, data set 7 , but only by a slight margin over the gamma. No distribution tested yielded non-zero critical levels for data set 6 .

Figures 4-7 show examples of histogram-pdf plots of typical data sets from each of the four groups of behavior

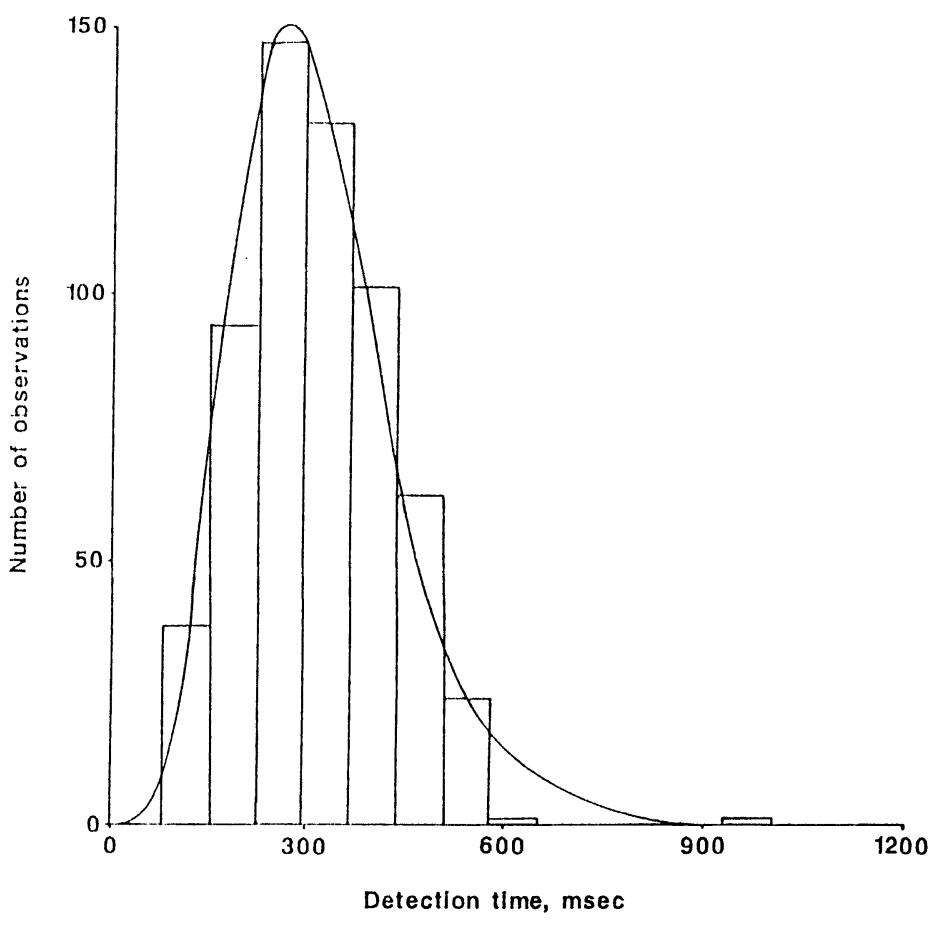

Fig. 4. Histogram of data set 1 with the gamma pdf. 
identified by the box plots. The shapes of these four histograms clearly support the results of the chi-square tests which suggest that the data are not exponentially distributed.

\section{TABLE 2}

Results of Chi-Square Tests of Goodness of Fit: Value of Test Statistic, (Number of Intervals), Critical Value - shown under the value of the test statistic.

(Blank entries represent tests with critical levels of zero.)

\begin{tabular}{cllc}
\hline $\begin{array}{c}\text { Set } \\
\#\end{array}$ & Weibull & Gamma & Lognormal \\
\hline 1 & $8.12(5)$ & $8.56(7)$ & $11.6(4)$ \\
& $1.7 \mathrm{E}-2$ & $7.3 \mathrm{E}-2$ & $6.7 \mathrm{E}-4$ \\
2 & $4.92(4)$ & $4.17(4)$ & $4.61(4)$ \\
& $2.7 \mathrm{E}-2$ & $4.1 \mathrm{E}-2$ & $3.1 \mathrm{E}-2$ \\
3 & $13.6(7)$ & $5.43(4)$ & $9.80(4)$ \\
& $8.7 \mathrm{E}-2$ & $2.0 \mathrm{E}-2$ & $1.7 \mathrm{E}-3$ \\
4 & $1.43(4)$ & $.973(4)$ & $19.0(6)$ \\
& $2.3 \mathrm{E}-1$ & $3.2 \mathrm{E}-1$ & $2.8 \mathrm{E}-4$ \\
5 & $3.16(4)$ & $2.24(4)$ & $13.7(8)$ \\
& $7.5 \mathrm{E}-2$ & $1.3 \mathrm{E}-1$ & $1.8 \mathrm{E}-2$
\end{tabular}

6

\begin{tabular}{|c|c|c|c|}
\hline 7 & $\begin{array}{c}14.7 \quad(8) \\
1.2 \mathrm{E}-2\end{array}$ & $\begin{array}{l}3.05 \quad(4) \\
8.1 \mathrm{E}-2\end{array}$ & $\begin{array}{l}2.92(4) \\
8.7 \mathrm{E}-2\end{array}$ \\
\hline 8 & $\begin{array}{c}14.7 \quad(10) \\
4.1 \mathrm{E}-2\end{array}$ & $\begin{array}{c}13.3 \quad(8) \\
2.0 \mathrm{E}-2\end{array}$ & $\begin{array}{c}20.7 \text { (9) } \\
2.1 \mathrm{E}-3\end{array}$ \\
\hline 9 & $\begin{array}{l}3.29 \quad(4) \\
7.0 \mathrm{E}-2\end{array}$ & $\begin{array}{l}2.04 \quad(4) \\
1.5 \mathrm{E}-1\end{array}$ & $\begin{array}{c}11.4 \quad(4) \\
7.3 \mathrm{E}-4\end{array}$ \\
\hline 10 & $\begin{array}{l}2.49 \text { (4) } \\
1.1 \mathrm{E}-1\end{array}$ & $\begin{array}{l}2.73 \quad(4) \\
9.8 \mathrm{E}-2\end{array}$ & $\begin{array}{l}9.57(4) \\
2.0 \mathrm{E}-3\end{array}$ \\
\hline 1 & $\begin{array}{l}6.88 \quad(5) \\
3.2 \mathrm{E}-2\end{array}$ & $\begin{array}{l}8.18 \quad(6) \\
4.2 \mathrm{E}-2\end{array}$ & $\begin{array}{c}16.2(9) \\
1.3 \mathrm{E}-2\end{array}$ \\
\hline & $\begin{array}{cc}27.4 \quad(6) \\
4.8 \mathrm{E}-6\end{array}$ & $\begin{array}{cc}27.5 & (6) \\
4.6 \mathrm{E}-6\end{array}$ & $\begin{array}{c}30.0 \quad(5) \\
3.0 \mathrm{E}-7\end{array}$ \\
\hline & $\begin{array}{c}13.8 \quad(8) \\
1.7 \mathrm{E}-2\end{array}$ & $\begin{array}{c}25.2 \quad(4) \\
1.3 \mathrm{E}-6\end{array}$ & \\
\hline & $\begin{array}{c}17.4 \quad(6) \\
6.0 \mathrm{E}-4\end{array}$ & $\begin{array}{c}12.2 \text { (7) } \\
1.6 \mathrm{E}-2\end{array}$ & $\begin{array}{c}27.5 \quad(5) \\
1.1 \mathrm{E}-6\end{array}$ \\
\hline & $\begin{array}{c}10.3 \quad(6) \\
1.6 \mathrm{E}-2\end{array}$ & $\begin{array}{c}11.2 \text { (7) } \\
2.4 \mathrm{E}-2\end{array}$ & $\begin{array}{c}20.0 \quad(7) \\
5.0 \mathrm{E}-4\end{array}$ \\
\hline & $\begin{array}{c}11.3 \quad(5) \\
3.6 \mathrm{E}-3\end{array}$ & $\begin{array}{cc}14.3 \quad(5) \\
7.8 \mathrm{E}-4\end{array}$ & $\begin{array}{c}16.22(4) \\
5.7 \mathrm{E}-5\end{array}$ \\
\hline & $\begin{array}{c}17.7 \quad(5) \\
1.5 \mathrm{E}-4\end{array}$ & $\begin{array}{c}32.3 \quad(8) \\
5.2 \mathrm{E}-6\end{array}$ & $\begin{array}{c}15.7 \quad(4) \\
7.7 \mathrm{E}-5\end{array}$ \\
\hline & $\begin{array}{c}13.6 \quad(7) \\
8.5 \mathrm{E}-3\end{array}$ & $\begin{array}{c}12.5 \text { (7) } \\
1.4 \mathrm{E}-2\end{array}$ & $\begin{array}{c}19.0 \quad(5) \\
7.8 \mathrm{E}-5\end{array}$ \\
\hline
\end{tabular}

The variation in shape indicated by the data suggests two possible approaches to parameter estimation. Either a family of distributions like the Weibull or gamma should be used to represent detection times or $s$-robust estimation methods which do not depend on a particular distribution may be necessary.

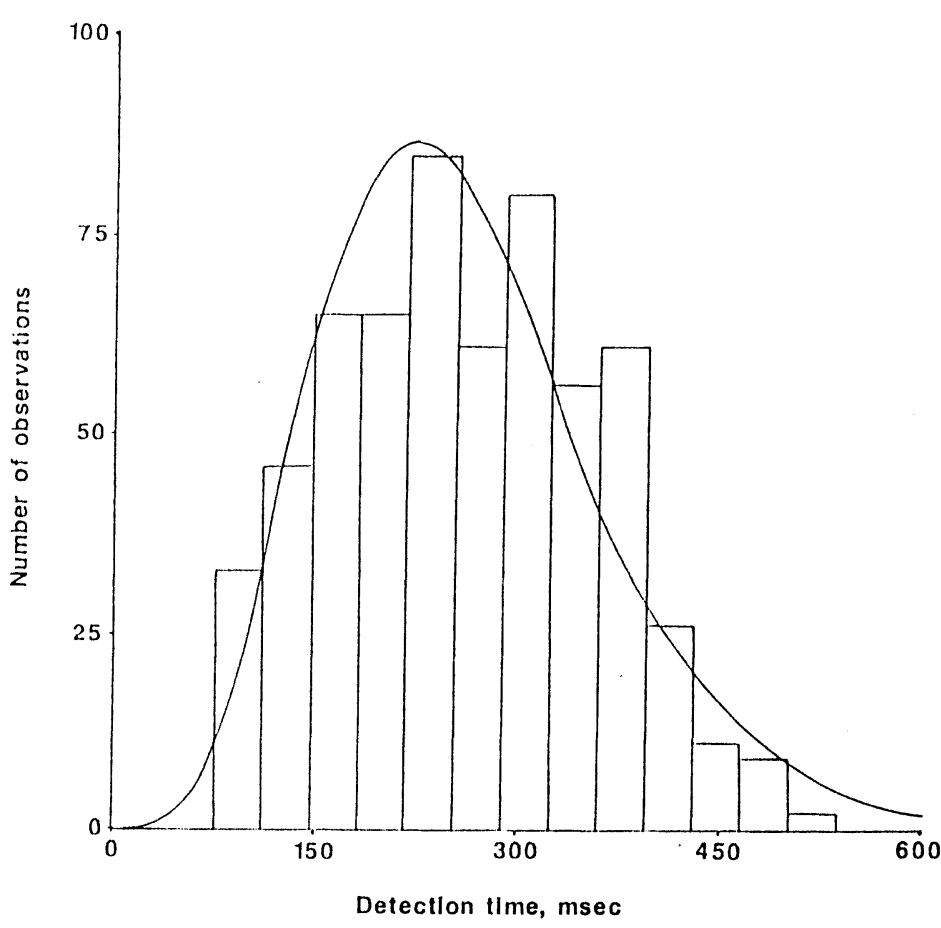

Fig. 5. Histogram of data set 9 with the gamma pdf.

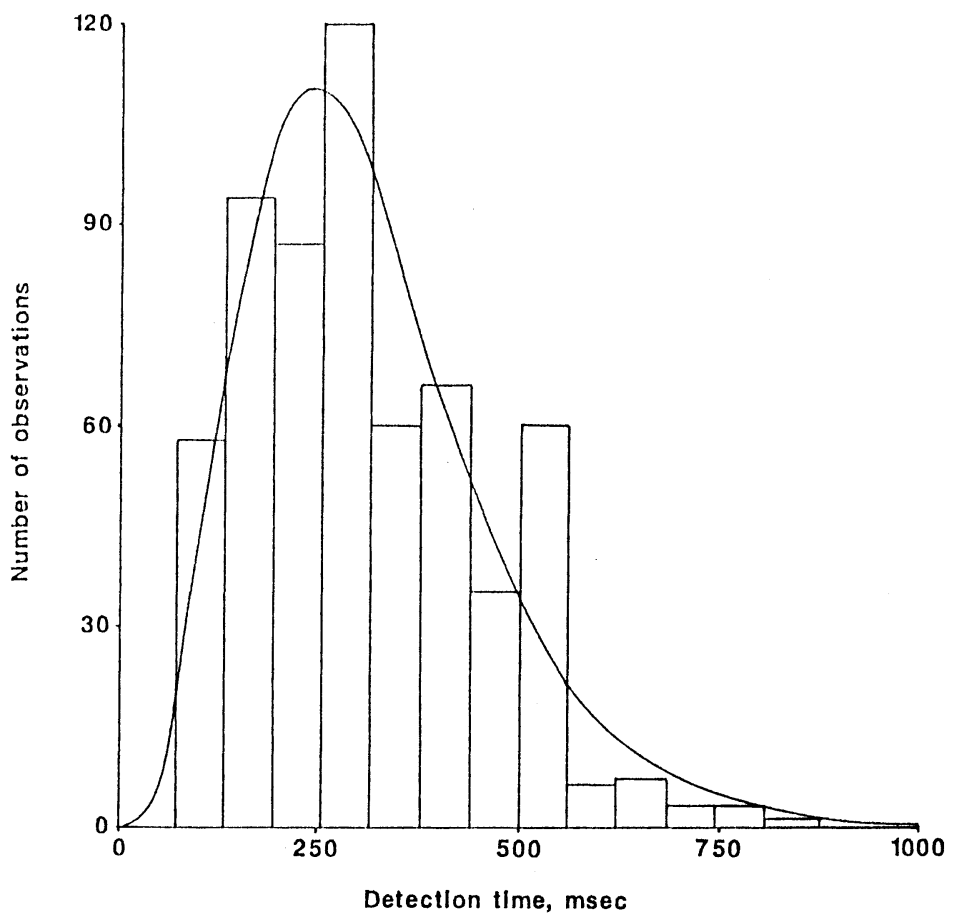

Fig. 6. Histogram of data set 11 with the gamma pdf.

\section{CONCLUDING REMARKS}

This work has shown that current sampling practices and common distributional assumptions may introduce unacceptable amounts of variation into reliability estimation. In the future, further fault injection experiments are planned. These experiments will seek to identify the sources of variation in detection behavior and investigate other 


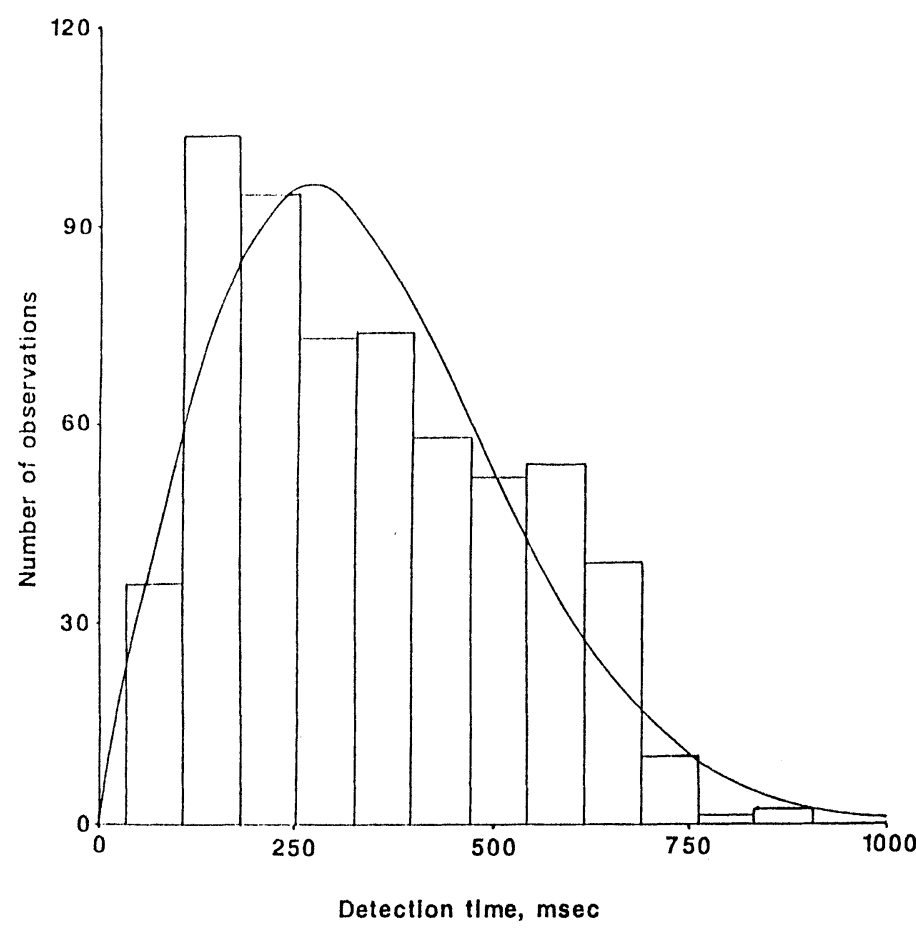

Fig. 7. Histogram of data set 16 with the Weibull pdf.

systems; the SIFT computer will also be subjected to fault injection. The results of these experiments will be used to develop stratified sampling methods which take advantage of grouping to increase the $s$-efficiency of parameter estimation. The new data will also be used to investigate $s$-robust techniques for parameter estimation.

\section{ACKNOWLEDGMENT}

I thank Carlos Liceaga, Peter Padilla, Frank Hill, Laura Wallace, and Murray Perry for conducting the fault injection on FTMP.

\section{REFERENCES}

[1] T. B. Smith III, J. H. Lala, "Development and evaluation of a fault-tolerant multiprocessor (FTMP) computer, volume IV, FTMP executive summary", NASA CR-172286, 1984 Feb.
[2] J. Goldberg, W. H. Kautz, P. M. Melliar-Smith, M. W. Green, K. N. Levitt, R. L. Schwartz, C. B. Weinstock, "Development and analysis of the software implemented fault-tolerance (SIFT) computer", NASA CR-172146, 1984 Feb.

[3] US MIL-HDBK-217D, Reliability Prediction of Electronic Equipment, 1982 Jan, Washington, DC, USA.

[4] H. M. Holt, A. O. Lupton, D. G. Holden, "Flight critical system design guidelines and validation methods", AIAA/AHS/ASEE Aircraft Design Systems and Operation Meeting, AIAA-84-2461, 1984.

[5] ORI Incorporated, "Validation methods for fault-tolerant avionics and control systems, working group meeting I", NASA CP-2114, 1979.

[6] Research Triangle Institute, "Validation methods for fault-tolerant avionics and control systems, working group meeting II", NASA CP-2130, 1980.

[7] N. R. Mann, R. E. Schafer, N. D. Singpurwalla, Methods for Statistical Analysis of Reliability and Life Data, John Wiley \& Sons, 1974.

[8] T. B. Smith III, J. H. Lala, "Development and evaluation of a fault-tolerant multiprocessor (FTMP) computer, volume III, FTMP test and evaluation", NASA CR-166073, 1983 May.

[9] T. B. Smith III, J. H. Lala, "Development and evaluation of a fault-tolerant multiprocessor (FTMP) computer, volume I, FTMP principles of operation", NASA CR-166071, 1983 May.

[10] V. Barnett, Elements of Sampling Theory, The English Universities Press Ltd., 1974, p 78.

[11] L. H. Koopmans, An Introduction to Contemporary Statistics, Duxbury Press, 1981.

[12] M. H. DeGroot, Probability and Statistics, Addison-Wesley Publishing Company, 1975, p 440.

[13] W. J. Conover, Practical Nonparametric Statistics, second edition, John Wiley \& Sons, 1980, p 80.

\section{AUTHOR}

George B. Finelli; Mail Stop 130; NASA Langley Research Center; Hampton Virginia 23665 USA.

George B. Finelli was born in 1956 and received a BA in computer science from the State University of New York at Buffalo in 1978, and a MS in statistics from Virginia Polytechnic Institute and State University in 1980. At VPI, he worked as a statistical consultant in the Statistical Consulting Lab. Since 1980, he has been an Aero-Space Technologist with the System Validation Methods Branch of the Information Systems Division at the NASA Langley Research Center. His areas of research include design and validation methods for fault-tolerant systems, performance and reliability modeling, data analysis, and statistical design of experiments. $\mathrm{He}$ is a member of the American Statistical Association.

Manuscript TR86-227 received 1986 May 31; revised 1987 January 2; revised 1987 April 20.

IEEE Log Number 15252 